\section{Variação do desempenho hospitalar segundo fontes de pagamento nos estados de São Paulo e Rio Grande do Sul, Brasil}

\author{
Variation in hospital performance according to \\ payment sources in the states of São Paulo and do \\ Rio Grande do Sul, Brazil
} Variación del desempeño hospitalario según
fuentes de pago en los estados de São Paulo y de
Río Grande do Sul, Brasil

\section{Resumo}

No Brasil, a convivência público-privado no financiamento e na prestação do cuidado ganha nítidos contornos na assistência hospitalar. Os arranjos de financiamento adotados pelos hospitais (Sistema Único de Saúde - SUS elou planos de saúde e/ou pagamento particular) podem afetar a qualidade do cuidado. Alguns estudos buscam associar a razão de mortalidade hospitalar padronizada (RMHP) a melhorias na qualidade. O objetivo foi analisar a RMHP segundo fonte de pagamento da internação e arranjo de financiamento do hospital. Analisaram-se dados secundários e causas responsáveis por $80 \%$ dos óbitos hospitalares. A RMHP foi calculada para cada hospital e fonte de pagamento. Hospitais com desempenho pior que o esperado (RMHP > 1) foram majoritariamente públicos de maior porte. A RMHP nas internações SUS foi superior, inclusive entre internações no mesmo hospital. Apesar dos limites, os achados indicam iniquidades no resultado do cuidado. Esforços voltados para a melhoria da qualidade de serviços hospitalares, independentemente das fontes de pagamento, são prementes.

Qualidade da Assistência à Saúde; Avaliação de Resultados (Cuidados de Saúde); Parcerias Público-Privadas; Mortalidade Hospitalar
Juliana Pires Machado 1

Mônica Martins 2

Iuri da Costa Leite 2

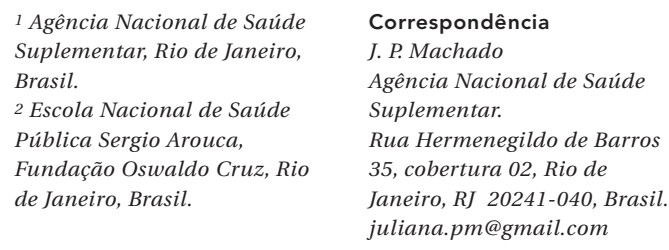

1 Agência Nacional de Saúde Suplementar, Rio de Janeiro, Brasil.

2 Escola Nacional de Saúde Pública Sergio Arouca,

Fundação Oswaldo Cruz, Rio de Janeiro, Brasil. 


\section{Introdução}

No Brasil, a convivência público-privado no âmbito do financiamento e da prestação dos cuidados em saúde ganha nítidos contornos na assistência hospitalar. Por um lado, é histórica a dependência do sistema público de saúde do cuidado hospitalar prestado por organizações privadas, com ou sem fins lucrativos. Por outro, a consolidação do mercado de planos de saúde privados ampliou a área de atuação do prestador privado. Nesse cenário, destaca-se a possibilidade de exclusividade ou sobreposição de clientelas pública e privada atendidas pelos mesmos hospitais 1,2 . As possíveis combinações entre as fontes de pagamento de internações adotadas pelos hospitais brasileiros (Sistema Único de Saúde - SUS, planos de saúde, e/ou pagamento particular), denominadas "arranjos de financiamento", indicam a clientela atendida e a estratégia para composição da reserva financeira, o que as caracterizam como elemento estrutural que pode afetar os resultados do cuidado 3 .

As medidas de qualidade da atenção hospitalar com foco no resultado do cuidado, especialmente a mortalidade hospitalar, destacamse quando são empregados apenas poucos indicadores, devido à sua capacidade de expressar o produto de todo o processo de cuidado 4,5,6. Entre as vantagens do uso da mortalidade hospitalar como medida de resultado do cuidado está o fato de a morte ser um evento definitivo e único, que, por lei, deve ser registrado e, portanto, espera-se melhor completude e acurácia no seu registro. Contudo, sabe-se que a relação entre o processo de cuidado e a morte não é inequívoca nem completamente compreendida 7. Isso impulsiona debates, ainda mais contundentes diante da sua aplicação para o pagamento por desempenho, e proposições metodológicas que permitam lidar com o impacto de fatores não relacionados à qualidade do cuidado sobre a mortalidade 8 .

Gradativamente, inúmeras comparações da mortalidade hospitalar foram desenvolvidas, com abordagens mais adequadas para minimizar o efeito dos fatores de confundimento, incluindo a seleção de causas especificas, o ajuste pelos fatores de risco do paciente (gravidade do caso) e as técnicas estatísticas empregadas 9,10,11,12. Do ponto de vista metodológico, a aplicação de técnicas de padronização de taxas considerando atributos relacionados à gravidade do paciente (ajuste de risco) busca algum controle sobre fatores que, independentemente do processo de cuidado, interferem sobre os resultados 13 . Nessa lógica, as variações residuais indicariam o efeito da qualidade da assistência prestada 13,14.
Outro ponto de destaque, refere-se à análise da mortalidade hospitalar global ou segundo problemas específicos, escolha marcada por controvérsias na literatura quanto à validade, utilidade para a tomada de decisão e finalidade da avaliação 15,16,17,18. Apoiados no incremento dos métodos e técnicas estatísticas para o ajuste de risco, na disponibilidade de grandes bases de dados administrativos e, sobretudo, no conhecimento disponível sobre a validade causal entre processo e resultado do cuidado, as análises da mortalidade hospitalar específica por doenças ou procedimentos cirúrgicos foram sendo mais exploradas 6,15,19. Apesar disso, o uso da mortalidade hospitalar global como medida para avaliação da variação entre hospitais é frequente nos programas de monitoramento de diversos países 15,20. O impulso no uso da mortalidade hospitalar global adveio do estudo de Jarman et al. 21, que comparou hospitais ingleses. Esses autores desenvolveram uma metodologia de cálculo da razão de mortalidade hospitalar padronizada (RMHP) com base em dados secundários, que prevê a inclusão de casos cujo diagnóstico principal que motivou a internação encontra-se entre as causas responsáveis por $80 \%$ das mortes hospitalares, e busca associar a variação da RMHP às características do prestador e da organização hospitalar. Isso constituiu um atrativo à sua aplicação em outros estudos e também por instituições governamentais na avaliação do efeito de inciativas para melhoria qualidade hospitalar na Holanda 22, no Canadá 7 e nos Estados Unidos 23.

Diversos estudos abordam a relação entre características estruturais e organizacionais dos hospitais e mortalidade hospitalar 24, destacando-se as análises sobre a natureza pública ou privada 2,25 e a fonte de pagamento da internação 26,27. Porém, a produção brasileira é ainda restrita 28 , e além disso o modelo para análise da mortalidade hospitalar global proposto por Jarman et al. ${ }^{21}$ ainda não foi aplicado em pacientes brasileiros.

Este estudo propôs investigar a relação das fontes de financiamento pública (SUS) e privada (não SUS) com o resultado do cuidado hospitalar prestado, explorando a mortalidade hospitalar global como medida de qualidade para avaliar o desempenho dos hospitais. Com essa perspectiva, busca-se contribuir para o entendimento das peculiaridades nacionais no tocante ao prestador público e privado de saúde e para o aprimoramento de metodologias que sirvam ao monitoramento da qualidade do cuidado hospitalar prestado no país. O objetivo principal foi analisar a variação da RMHP em função da fonte de pagamento da internação e do arranjo de financiamento do hospital. 


\section{Metodologia}

\section{Delineamento do estudo}

Estudo de corte transversal, realizado com base em dados secundários disponíveis nos sistemas de informação brasileiros. A mortalidade hospitalar foi selecionada como medida da qualidade do cuidado e do desempenho, tendo sido analisados somente os óbitos intra-hospitalares; tal recorte justifica-se pela factibilidade em face dos dados administrativos existentes. A abordagem metodológica para avaliação da mortalidade hospitalar global desenvolvida por Jarman et al. 21 foi aplicada para avaliar a variação entre prestadores de cuidado hospitalar financiados pelo SUS e por planos privados de saúde ou particular. Considerando a disponibilidade de informações nas bases de dados brasileiras uma adaptação da proposição de Jarman et al. 21,22 foi realizada com relação à seleção das variáveis incluídas no estudo.

\section{Fontes de dados}

O Sistema de Informações Hospitalares (SIH) foi utilizado como fonte de dados para as internações financiadas pelo SUS e a Comunicação de Informação Hospitalar e Ambulatorial (CIHA) para as internações não SUS (fonte de pagamento: plano de saúde ou particular). O Cadastro Nacional de Estabelecimentos de Saúde do Ministério da Saúde (CNES) e o Registro de Planos de Saúde da Agência Nacional de Saúde Suplementar (RPS/ANS) foram fonte de dados sobre a estrutura da rede hospitalar.

\section{Universo de estudo}

No Brasil, foram realizadas 39.419.539 internações no período de estudo (2008-2010), das quais $15,9 \%$ não foram financiadas pelo SUS. O universo de estudo está circunscrito às internações realizadas entre 2008 e 2010 em hospitais dos estados de São Paulo e Rio Grande do Sul. A delimitação desse recorte geográfico deve-se à maior cobertura da população por planos de saúde (acima da média nacional) e ao menor sub-registro no preenchimento dos sistemas de produção hospitalar 3. Dessas internações, foram selecionadas as referentes a pacientes com idades entre 18 e 99 anos, que permaneceram menos de 30 dias internados (internações agudas), não transferidos, ocorridas em hospitais com pelo menos uma internação-dia (ou seja, 1.095 internações ou mais nos três anos).

Em conformidade com a metodologia de Jarman et al. 21,22, foram excluídas internações cujo diagnóstico principal se referisse a grupos inespecíficos de causas, isto é, categorias diagnósticas cujo primeiro digito do código da Classificação Internacional de Doenças, 10a revisão (CID-10) eram "R", “T", "V", "X", "Y” ou "Z”, incluídas nos capítulos: "XVIII - Sintomas, sinais e achados anormais de exames clínicos e de laboratório, não classificados em outra parte"; "XIX - Lesões, envenenamento e algumas outras consequências de causas externas"; "XX - Causas externas de morbidade e de mortalidade"; e "XXI - Fatores que influenciam o estado de saúde e o contato com os serviços de saúde”. Em seguida, as internações foram filtradas por diagnóstico principal (códigos da CID-10 com 3 dígitos), incluindo somente as causas responsáveis por $80 \%$ dos óbitos hospitalares 21. Das 7.385.323 internações ocorridas em São Paulo e Rio Grande do Sul, ao final desse processo o universo de estudo incluiu 5.636.778 de casos.

Dentre os 5.636.778 de internações, as 16 categorias diagnósticas responsáveis por $50 \%$ das mortes registradas nas bases de dados brasileiras incluíram: pneumonia por micro-organismo não identificado; insuficiência cardíaca; outras septicemias; insuficiência respiratória não classificada em outra parte; acidente vascular cerebral não especificado como hemorrágico ou isquêmico; infarto agudo do miocárdio; outras doenças pulmonares obstrutivas crônicas; pneumonia bacteriana não classificada em outra parte; traumatismo craniano; insuficiência renal crônica outras doenças do aparelho digestivo; neoplasia maligna dos brônquios e dos pulmões; angina pectoris; infecção bacteriana de localização não especificada; hemorragia intracerebral; e desnutrição proteico-calórica não especificada. Somadas a outras 59 causas, 75 categorias diagnósticas responderam por $80 \%$ das mortes hospitalares registradas no período.

\section{Análise dos dados}

As variáveis independentes centrais deste estudo foram as fontes de pagamento das internações (SUS e não SUS) e os arranjos de financiamento (SUS; Não SUS - plano de saúde ou particular; Misto - SUS e plano de saúde ou particular) adotados pelos hospitais; em que fonte de pagamento está no nível da internação e arranjo de financiamento no nível do hospital. Nos arranjos "Não SUS" e "Misto" incluíram-se também alguns casos com fonte de pagamento filantropia. Outras características da estrutura da rede hospitalar (natureza jurídica e porte) e características do processo de cuidado (uso de unidade de terapia intensiva, tempo de permanência e tipo de procedimento realizado) foram também analisadas. 
Modelo de ajuste de risco

A variável dependente: RMHP pressupõe o cálculo dos óbitos esperados em função dos fatores de risco no nível de cada internação. Diversos modelos de ajuste de risco para predição do óbito hospitalar foram testados. Utilizando a regressão logística tradicional (óbito sim/não) foram incluídas no modelo de base (composto por idade como variável categórica: 18-49, 50$59,60-69,70-79,80-89$ e 90-99 anos, e sexo dicotômica), passo a passo, as seguintes variáveis: diagnóstico principal entre as causas responsável por $50 \%$ dos óbitos hospitalares ( $\operatorname{sim} /$ não); escore do índice de Charlson (IC) 29 aplicado ao diagnóstico secundário - comorbidade (variável categórica: $0,1,2$ e $\geq 3$ ) e ao diagnóstico principal (dicotômica); presença de cada uma das comorbidades de Elixhauser estatisticamente significante e não incluída no IC (sim/não) 29; presença de comorbidade (sim/não). Para os índices de comorbidade (Charlson e Elixhauser) foi utilizado o algoritmo para codificação na CID-10 proposto por Quan et al. ${ }^{30}$. Optou-se por utilizar dois índices de comorbidade e a presença simples de comorbidade pela insuficiência de campo para o registro de diagnóstico secundário e subnotificação dessa informação nas fontes de dados utilizadas. O IC foi calculado não só para a comorbidade, mas também para o diagnóstico principal, por causa do número de categorias diagnósticas (75 categorias) e sua heterogeneidade. Além disso, testou-se a inclusão da variável para indicar se o diagnóstico principal estava entre as causas responsáveis por $50 \%$ das mortes, mantendo coerência com a proposta de Jarman et al. 21. As variáveis aqui testadas são semelhantes às incorporadas no ajuste de risco em vários estudos, especialmente naqueles baseados em dados administrativos 4,13,26,27. Daquelas amplamente empregadas, apenas o tipo de admissão (emergência/eletiva) não pôde ser utilizado, por indisponibilidade no CIHA.

A capacidade de discriminação do modelo de risco, incluindo as variáveis acima relacionadas, foi julgada com base na estatística C (Receiver Operating Characteristics - ROC curve scores). Esse teste mede a probabilidade do risco de morte em um paciente selecionado aleatoriamente e que morreu, comparada à probabilidade do risco de morte em um paciente selecionado aleatoriamente e que sobreviveu. Valores inferiores a 0,7 implicam pobre discriminação, entre 0,7 e 0,8 razoável, e acima de 0,8 considera-se o modelo preditivo de boa discriminação ${ }^{31}$. A capacidade preditiva do modelo de risco final obtida foi 0,795 (IC95\%: 0,795-0,796), agregando poder de discriminação comparativamente ao modelo de base (idade e sexo) cuja estatística C era 0,720 (IC95\%: 0,720-0,721). O pacote estatístico utilizado foi o SPSS (SPSS Inc., Chicago, Estados Unidos).

\section{Avaliação da mortalidade hospitalar}

Com base no somatório das probabilidades de morte preditas para cada internação estimadas pela regressão logística, foi calculado o número esperado de mortes (E) em cada hospital. Assim, a razão de mortalidade hospitalar padronizada (RMHP: O/E) é produto divisão do número de mortes observadas $(\mathrm{O})$ pelo número de mortes esperadas (E). A ordenação da RMHP foi utilizada para classificar os hospitais estudados segundo seu desempenho, adotando-se o corte em percentis proposto por Noronha et al. 32: razão O/E no percentil 20 - desempenho melhor que o esperado; razão $\mathrm{O} / \mathrm{E}$ entre percentis 20 e 80 desempenho conforme esperado; e razão $\mathrm{O} / \mathrm{E}$ no percentil 80 - desempenho pior que o esperado.

Essa etapa da análise incluiu apenas os hospitais que registraram pelo menos uma morte no período estudado e cuja RMHP estava no intervalo de confiança de até 3 desvios-padrão abaixo ou acima da média, tendo por justificativa a melhoria das análises de dispersão e a exclusão de casos desviantes. Dos 760 hospitais incluídos no universo de estudo, 14 não registraram óbito no período analisado. Nesses, ocorreram 49.036 internações, com variação entre 1.268 e 9.867 internações por hospital, e média de 3.503 internações. Tratava-se de hospitais de menor porte, majoritariamente privados com fins lucrativos, de arranjo de financiamento Não SUS. Sequencialmente, entre os 746 hospitais retidos (registro mínimo de 1 óbito), 3 apresentaram RMHP maior que o limite superior (3 desvios- padrão acima da média) e foram excluídos, sendo considerados "desviantes". Esses três hospitais totalizaram 12.444 internações, eram de menor porte, localizavam-se no Estado de São Paulo, em municípios com mais de $30 \%$ da população coberta por plano de saúde, eram privados com fins lucrativos e com arranjo de financiamento Não SUS.

Para analisar especificamente o desempenho de hospitais cujo arranjo de financiamento era misto (SUS, planos e particular), a RMHP foi estratificada segundo fontes de pagamento da internação dentro desses hospitais, ou seja, foram calculadas as razões $(\mathrm{O} / \mathrm{E})$ nas internações financiadas pelo SUS e pelos planos privados; hospitais sem registro de pelo menos um óbito para cada fonte de pagamento foram excluídos. Nessa fase, admite-se alguma imprecisão por utilizar a mortalidade esperada calculada com base no total de internações. 
O estudo foi aprovado pelo Comitê de Ética em Pesquisa da Escola Nacional de Saúde Pública Sergio Arouca da Fundação Oswaldo Cruz ENSP/Fiocruz (Certificado de Apresentação para Apreciação Ética no 02234312.3.0000.5240; parecer no 78617, de 23 de agosto de 2012).

\section{Resultados}

Os 743 hospitais avaliados quanto ao seu desempenho registraram 5.575.298 internações (99\% do total), tendo em média 7.503 internações cada um nos três anos estudados. A taxa bruta de mortalidade hospitalar correspondeu a 5,8\%, variando entre 0 e 19\%; a taxa de mortalidade esperada foi de $6 \%$, variando entre 1,3 e $13 \%$ (Tabela 1). A RMHP variou entre 0 e 2,4\% (Tabela 1), foi menor que 1 em 463 hospitais, igual a 1 em 46, e maior que 1 em 234 hospitais (Tabela 1; Figura 1).

A classificação dos hospitais segundo os percentis 20 e 80 da RMHP identificou 165 hospitais com melhor desempenho que o esperado (percentil 20); eles registraram quase 897 mil internações, com pouco mais de 13 mil mortes, sendo a taxa de mortalidade bruta $1,5 \%$ e a esperada $4,9 \%$ (Tabela 1). Já os hospitais com desempenho pior que o esperado (percentil 80) realizaram mais de 1,6 milhão de internações, ocorrendo cerca de 156 mil mortes, com taxa de mortalidade bruta de $9,6 \%$ e esperada $6,4 \%$ (Tabela 1 ).

Dos 165 hospitais com melhor desempenho, a maioria localizava-se em São Paulo (67\%), 7 eram públicos, 100 privados sem fins lucrativos e 58 privados com fins lucrativos (Tabela 2). A maioria desses hospitais tinha arranjo de financiamento Misto (111) e porte menor ou igual a 49 leitos (68). Entre os pacientes internados nesses hospitais, a idade média foi 51 anos (menor que nos demais grupos) e havia maior registro de comorbidade e maior percentual de casos com escore diferente de zero nos dois índices de comorbidade (IC e Elixhauser). Nesses hospitais, foi encontrado menor uso de UTI $(3,7 \%)$ e maior proporção de procedimentos cirúrgicos $(54,3 \%)$ (Tabela 2). O diagnóstico principal referia menor proporção $(16,8 \%)$ de causas responsáveis por $50 \%$ das mortes, o IC aplicado ao diagnóstico principal apresentou maior proporção de casos igual a zero $(84,9 \%)$, indicando menor risco nesse grupo associado à doença que motivou a internação. Predominava o pagamento da internação por planos de saúde, enquanto nos outros percentis o SUS era a maior fonte de pagamento (Tabela 2).

A distribuição da RMHP por fontes de pagamento e arranjos de financiamento apresentou grande variação (Figura 2). A RMHP nas interna- ções financiadas pelo SUS foi em geral superior àquela verificada para os demais pacientes em todos os arranjos de financiamento. As maiores discrepâncias foram observadas nas internações de hospitais com arranjo Misto, em que a razão foi de 1,1 para os pacientes do SUS, 0,6 para pacientes de planos privados de saúde e 0,5 para os particulares (Figura 2). A menor RMHP foi verificada entre as internações por planos ou particulares em hospitais com arranjo misto $(0,5)$, enquanto as maiores estavam nas internações SUS e filantropia, em hospitais com arranjo somente SUS $(1,4)$ (Figura 2).

Entre os 536 hospitais com arranjo misto, 362 registraram pelo menos uma morte entre internações financiadas pelo SUS e por plano de saúde (Tabela 3). Em apenas 57 hospitais a RMHP foi menor nos pacientes do SUS do que naqueles de planos (284 mil internações), enquanto nos demais hospitais (305) a razão para pacientes do SUS foi superior àquela verificada para pacientes de planos (2,5 milhões de internações). A variação da RMHP no interior desses hospitais mantém o resultado desfavorável para os pacientes no SUS: RMHP entre $0,13-2,19$ no SUS versus 0,02-1,69 entre os pacientes dos planos de saúde (Tabela 3).

\section{Discussão}

A metodologia proposta por Jarman et al. 21, apesar das adaptações realizadas neste estudo, apresentou razoável capacidade discriminativa do modelo de risco $(C=0,795)$, sendo limítrofe a faixa classificada como boa (a partir de 0,8) 31 . Na discussão sobre vantagens e problemas do uso da mortalidade hospitalar global ou específica por condição, é importante ter em conta que análises por condições específicas utilizando dados administrativos brasileiros vinham apresentando capacidade discriminativa dos modelos de risco inadequadas (estatística C inferior a 0,70) 3,29. A inclusão de 75 categorias diagnósticas na análise de $80 \%$ dos óbitos intra-hospitalares mostrou-se compatível com o resultado de estudos realizados na Inglaterra 21 e Canadá 7 , onde os óbitos concentravam-se em 85 e 65 diagnósticos, respectivamente. É complexa a comparação com os estudos dos Estados Unidos 23 e Holanda 22, uma vez que nesses estudos não foi utilizada a CID-10, mas sim a codificação por CCS (Clinical Classification System) da Agency for Healthcare Research and Quality dos Estados Unidos (AHRQ), com base na ICD-9-CM. Quanto à variável indicativa de gravidade, baseada nos diagnósticos responsáveis por $50 \%$ dos óbitos, neste estudo 34 causas foram selecionadas, com menor concentração 
Classificação do desempenho dos hospitais, segundo a razão de mortalidade hospitalar padronizada (RMHP). Estados de São Paulo e Rio Grande do Sul, Brasil, 2008-2010.

\begin{tabular}{|c|c|c|c|c|}
\hline \multirow[t]{2}{*}{ Variáveis } & \multirow[t]{2}{*}{ Total } & \multicolumn{3}{|c|}{ Percentil } \\
\hline & & 20 * & $30-70 * \star$ & $80 * \star \star$ \\
\hline \multicolumn{5}{|l|}{ Hospitais } \\
\hline $\mathrm{n}$ & 743 & 165 & 398 & 180 \\
\hline \multicolumn{5}{|l|}{ Internações } \\
\hline $\mathrm{n}$ & 5.575 .298 & 896.830 & 3.051 .050 & 1.627 .418 \\
\hline Variação & $1.041-59.902$ & $1.041-33.673$ & $1.117-59.902$ & $1.212-45.191$ \\
\hline Média & 7.503 & 5.435 & 7.665 & 9.041 \\
\hline \multicolumn{5}{|l|}{ Mortes observadas } \\
\hline $\mathrm{n}$ & 325.470 & 13.297 & 156.443 & 155.730 \\
\hline Variação & $1-4.421$ & $1-689$ & $29-4.222$ & $89-4.421$ \\
\hline Média & 438 & 81 & 393 & 865 \\
\hline \multicolumn{5}{|l|}{ Mortes esperadas } \\
\hline $\mathrm{n}$ & 332.894 & 43.893 & 185.434 & 103.567 \\
\hline Variação & $29-3.763$ & $29-2.060$ & $49-3.763$ & $71-2.911$ \\
\hline Média & 448 & 266 & 466 & 575 \\
\hline \multicolumn{5}{|c|}{ Taxa de mortalidade bruta } \\
\hline$\%$ & 5,8 & 1,5 & 5,1 & 9,6 \\
\hline Variação & $0,0-19,1$ & $0,0-4,1$ & $1,3-10,8$ & $4,5-19,1$ \\
\hline \multicolumn{5}{|c|}{ Taxa de mortalidade esperada } \\
\hline$\%$ & 6,0 & 4,9 & 6,1 & 6,4 \\
\hline Variação & $1,3-13,1$ & $1,3-13,1$ & $2,5-12,9$ & $3,0-12,1$ \\
\hline Desvio padrão & 1,7 & 2,2 & 1,5 & 1,5 \\
\hline \multicolumn{5}{|l|}{ RMHP (O/E) } \\
\hline$\%$ & 1,0 & 0,3 & 0,8 & 1,5 \\
\hline Variação & $0,0-2,4$ & $0,0-0,4$ & $0,5-1,1$ & $1,2-2,4$ \\
\hline Desvio padrão & 0,5 & 0,1 & 0,2 & 0,3 \\
\hline
\end{tabular}

* Desempenho melhor que o esperado;

** Desempenho compatível ao esperado;

$\star \star \star$ Desempenho pior que o esperado.

Fonte: Ministério da Saúde. Cadastro Nacional de Estabelecimentos de Saúde (CNES), Registro de Planos de Saúde (RPS),

Sistema de Informações Hospitalares do SUS (SIH) e Comunicação de Internação Hospitalar (CIH).

do que a obtida na base inglesa, na qual 15 causas formavam esse grupo 21 .

Apesar da sua atratividade em função da característica global e boa capacidade preditiva, a seleção de causas baseada na proposta de Jarman et al. 21 é considerada mais imprecisa, já que a inclusão de muitos e variados motivos implica maior heterogeneidade do risco, sendo o tratamento do diagnóstico principal no modelo de risco mais complexo, o que pode levantar questionamentos quanto à validade atribuível 17,18. Entretanto, essa ferramenta pode auxiliar o monitoramento temporal e assinalar problemas ou melhorias em ações voltadas para garantir a qualidade do cuidado hospitalar, tanto para os gestores das unidades quanto para gestores da rede.

Quanto ao ajuste de risco realizado, as variáveis utilizadas neste estudo guardam similaridades com outros trabalhos 4,14,26,27,31. Nos estudos nacionais, a idade, o sexo, a utilização de UTI, o tipo de admissão e o diagnóstico principal têm sido as principais variáveis usadas para a padronização de taxas e modelos de ajuste de risco, pois se encontram disponíveis nas bases brasileiras 28,29,33,34. A informação sobre comorbidade e os índices dela derivados têm sido menos empregados, especialmente por conta do sub-registro e da incompletude dos dados de diagnóstico secundários 28,29 . 
Variação da razão de mortalidade hospitalar padronizada (observada/esperada - razão O/E). Estados de São Paulo e Rio Grande do Sul, Brasil, 2008-2010.

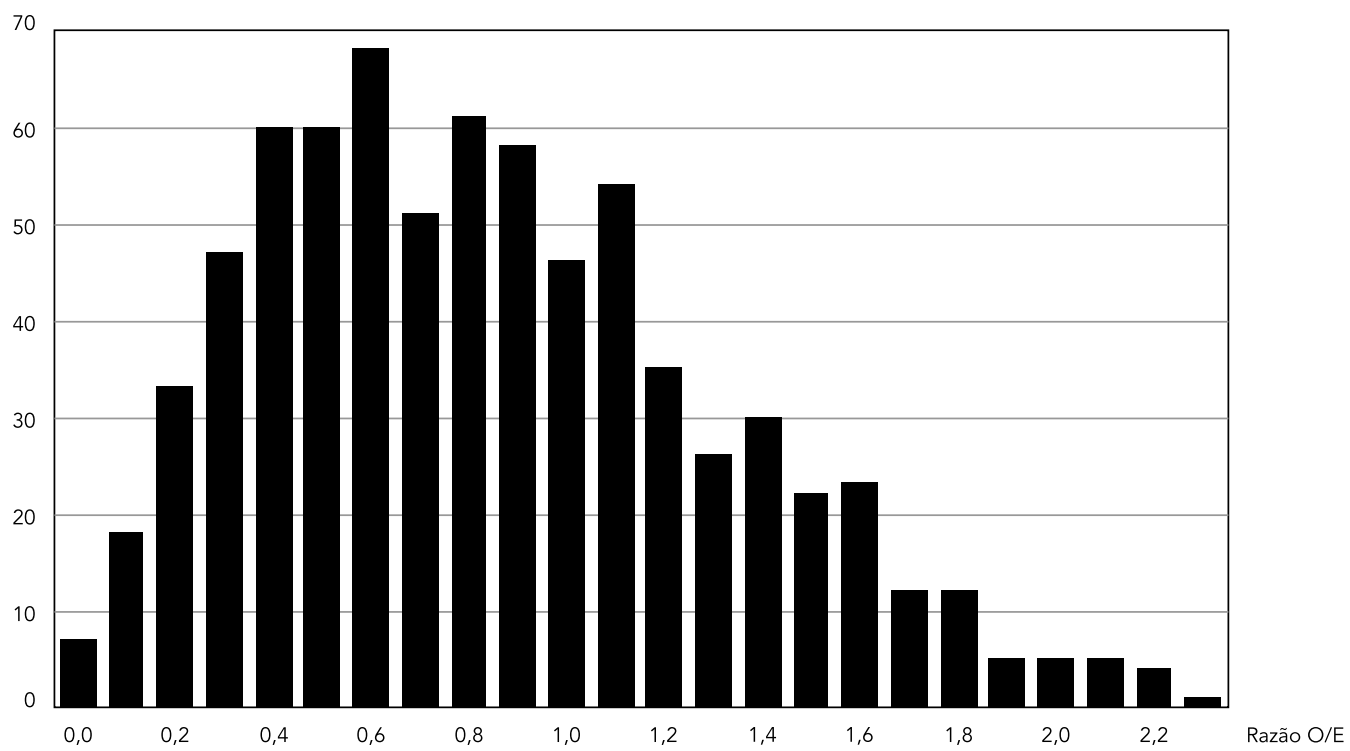

Para lidar com a variação das práticas de alta adotadas pelos hospitais, Jarman et al. 21 utilizaram o tempo de permanência como variável de ajuste da mortalidade hospitalar, a exemplo de alguns estudos pioneiros 35 . Outros estudos não costumam incluir esta variável, mas sim considerá-la uma medida relacionada ao processo de cuidado e à eficiência técnica 9,36. A discussão a respeito do significado de determinado padrão do tempo de permanência é complexa, uma vez que seu efeito sobre a mortalidade é ambíguo e os seus determinantes são diversos. Além dos motivos clínicos, há práticas gerenciais voltadas para a maior eficiência, que podem estimular a redução no tempo de permanência, e gerar inclusive altas precoces. A disponibilidade de leitos de longa permanência ou unidades de reabilitação também pode afetar o tempo de permanência e não representar necessariamente inadequações no processo de cuidado 19,35,36. Por outro lado, as mortes ocorridas no primeiro dia de internação podem decorrer da gravidade do caso, barreiras de acesso ou inadequação do cuidado de emergência. Considerando a dificuldade de separar complicação, gravidade e adequação do cuidado na explicação do tempo de permanência e do seu efeito sobre a mortalidade hospitalar, no presente trabalho, diferente de Jarman et al. 21, optou-se por excluir o tempo de permanência do modelo de ajuste de risco. Destaca-se, contudo, que a análise aqui realizada se deteve sobre as internações até 30 dias, buscando privilegiar a relação entre processo de cuidado e óbito, levando em conta que quanto mais distante temporalmente o óbito menor sua associação com o processo de cuidado recebido 13 .

Ainda que não tenham sido explorados outros aspectos de natureza organizacional e financeira tidos como relacionados à qualidade, tais como o contexto e cultura institucional, diferenças regionais e o grau de atuação de mercados privados 25 , entre as características estruturais dos hospitais estudados o desempenho relativamente melhor de hospitais de pequeno porte se destacou, achado que parece mais associado ao perfil menos complexo dos casos, indicado tanto pelas variáveis descritivas do risco do paciente como pelo menor uso de UTI, o que também pode se relacionar à menor disponibilidade desses leitos.

Especificamente em relação à análise das fontes de pagamento da assistência hospitalar brasileira, há alguns estudos sobre a composição público-privada da assistência e dos gastos hospitalares nacionais, enfocando as perspectivas política, econômica, administrativa e do 
Tabela 2

Características dos pacientes, do processo de cuidado e da estrutura dos hospitais classificados por percentis de desempenho. Estados de São Paulo e Rio Grande do Sul, Brasil, 2008-2010.

\begin{tabular}{|c|c|c|c|c|c|c|c|c|}
\hline \multirow[t]{3}{*}{ Variáveis } & \multirow{2}{*}{\multicolumn{2}{|c|}{ Total }} & \multicolumn{6}{|c|}{ Percentis } \\
\hline & & & \multicolumn{2}{|c|}{20 * } & \multicolumn{2}{|c|}{$30-70 * *$} & \multicolumn{2}{|c|}{$80 \star \star \star$} \\
\hline & $\mathrm{n}$ & $\%$ & $\mathbf{n}$ & $\%$ & n & $\%$ & n & $\%$ \\
\hline \multicolumn{9}{|l|}{ Hospital } \\
\hline Total & 743 & 100,0 & 165 & 100,0 & 398 & 100,0 & 180 & 100,0 \\
\hline \multicolumn{9}{|l|}{ UF } \\
\hline São Paulo & 537 & 72,3 & 111 & 67,3 & 277 & 69,6 & 149 & 82,8 \\
\hline Rio Grande do Sul & 206 & 27,7 & 54 & 32,7 & 121 & 30,4 & 31 & 17,2 \\
\hline \multicolumn{9}{|l|}{ Natureza } \\
\hline Público & 148 & 19,9 & 7 & 4,2 & 50 & 12,6 & 91 & 50,6 \\
\hline Privado sem fins lucrativos & 451 & 60,7 & 100 & 60,6 & 269 & 67,6 & 82 & 45,6 \\
\hline Privado com fins lucrativos & 144 & 19,4 & 58 & 35,2 & 79 & 19,8 & 7 & 3,9 \\
\hline \multicolumn{9}{|l|}{ Arranjo } \\
\hline Somente SUS & 83 & 11,2 & 5 & 3,0 & 24 & 6,0 & 54 & 30,0 \\
\hline Misto (SUS, planos e particular) & 533 & 71,7 & 111 & 67,3 & 301 & 75,6 & 121 & 67,2 \\
\hline Não SUS (planos e particular) & 127 & 17,1 & 49 & 29,7 & 73 & 18,3 & 5 & 2,8 \\
\hline \multicolumn{9}{|l|}{ Porte (leitos) } \\
\hline$\leq 49$ & 162 & 21,8 & 68 & 41,2 & 80 & 20,1 & 14 & 7,8 \\
\hline $50-149$ & 355 & 47,8 & 63 & 38,2 & 216 & 54,3 & 76 & 42,2 \\
\hline $150-299$ & 176 & 23,7 & 30 & 18,2 & 74 & 18,6 & 72 & 40,0 \\
\hline$\geq 300$ & 50 & 6,7 & 4 & 2,4 & 28 & 7,0 & 18 & 10,0 \\
\hline \multicolumn{9}{|c|}{ Cobertura por plano de saúde do município (\%) } \\
\hline $0,1-5,0$ & 48 & 6,5 & 16 & 9,7 & 30 & 7,5 & 2 & 1,1 \\
\hline $5,1-10,0$ & 88 & 11,8 & 30 & 18,2 & 47 & 11,8 & 11 & 6,1 \\
\hline $10,1-20,0$ & 127 & 17,1 & 25 & 15,2 & 77 & 19,3 & 25 & 13,9 \\
\hline $20,1-30,0$ & 104 & 14,0 & 21 & 12,7 & 55 & 13,8 & 28 & 15,6 \\
\hline Mais de 30,0 & 376 & 50,6 & 73 & 44,2 & 189 & 47,5 & 114 & 63,3 \\
\hline \multicolumn{9}{|l|}{ Paciente } \\
\hline Total & 5.575 .298 & 100,0 & 896.830 & 100,0 & 3.051 .050 & 100,0 & 1.627 .418 & 100,0 \\
\hline \multicolumn{9}{|l|}{ Sexo } \\
\hline Homens & 2.635 .376 & 47,3 & 375.645 & 41,9 & 1.437 .814 & 47,1 & 821.917 & 50,5 \\
\hline Mulheres & 2.939 .922 & 52,7 & 521.185 & 58,1 & 1.613 .236 & 52,9 & 805.501 & 49,5 \\
\hline Idade (média) & 54 & - & 51 & - & 55 & - & 54 & - \\
\hline \multicolumn{9}{|l|}{ Faixa etária (anos) } \\
\hline $40-49$ & 2.342 .557 & 42,0 & 447.645 & 49,9 & 1.234 .797 & 40,5 & 660.115 & 40,6 \\
\hline $50-59$ & 967.290 & 17,3 & 142.569 & 15,9 & 526.713 & 17,3 & 298.008 & 18,3 \\
\hline $60-69$ & 896.169 & 16,1 & 120.905 & 13,5 & 499.753 & 16,4 & 275.511 & 16,9 \\
\hline $70-79$ & 810.430 & 14,5 & 108.434 & 12,1 & 465.969 & 15,3 & 236.027 & 14,5 \\
\hline $80-89$ & 467.509 & 8,4 & 64.576 & 7,2 & 271.376 & 8,9 & 131.557 & 8,1 \\
\hline $90-99$ & 91.343 & 1,6 & 12.701 & 1,4 & 52.442 & 1,7 & 26.200 & 1,6 \\
\hline \multicolumn{9}{|l|}{ Comorbidade } \\
\hline Não & 4.489 .584 & 80,5 & 644.242 & 71,8 & 2.562 .798 & 84,0 & 1.282 .544 & 78,8 \\
\hline Sim & 1.085 .714 & 19,5 & 252.588 & 28,2 & 488.252 & 16,0 & 344.874 & 21,2 \\
\hline \multicolumn{9}{|l|}{ IC } \\
\hline 0 & 5.466 .272 & 98,0 & 876.666 & 97,8 & 3.001 .300 & 98,4 & 1.588 .306 & 97,6 \\
\hline 1 & 68.977 & 1,2 & 14.536 & 1,6 & 31.341 & 1,0 & 23.100 & 1,4 \\
\hline 2 & 29.965 & 0,5 & 4.253 & 0,5 & 14.041 & 0,5 & 11.671 & 0,7 \\
\hline$\geq 3$ & 10.084 & 0,2 & 1.375 & 0,2 & 4.368 & 0,1 & 4.341 & 0,3 \\
\hline
\end{tabular}

(continua) 
Tabela 2 (continuação)

\begin{tabular}{|c|c|c|c|c|c|c|c|c|}
\hline \multirow[t]{3}{*}{ Variáveis } & \multicolumn{2}{|c|}{ Total } & \multicolumn{6}{|c|}{ Percentis } \\
\hline & \multirow[b]{2}{*}{$\mathbf{n}$} & \multirow[b]{2}{*}{$\%$} & \multicolumn{2}{|c|}{20 * } & \multicolumn{2}{|c|}{$30-70 * \star$} & \multicolumn{2}{|c|}{$80 * * *$} \\
\hline & & & $\mathbf{n}$ & $\%$ & n & $\%$ & $\mathbf{n}$ & $\%$ \\
\hline \multicolumn{9}{|c|}{ Comorbidades de Elixhauser } \\
\hline 0 & 5.332 .700 & 95,6 & 837.946 & 93,4 & 2.938 .458 & 96,3 & 1.556 .296 & 95,6 \\
\hline 1 & 242.598 & 4,4 & 58.884 & 6,6 & 112.592 & 3,7 & 71.122 & 4,4 \\
\hline \multicolumn{9}{|c|}{ Diagnóstico principal entre os $50 \%$ mais fatais } \\
\hline Não & 4.158 .328 & 74,6 & 745.876 & 83,2 & 2.255 .846 & 73,9 & 1.156 .606 & 71,1 \\
\hline Sim & 1.416 .970 & 25,4 & 150.954 & 16,8 & 795.204 & 26,1 & 470.812 & 28,9 \\
\hline \multicolumn{9}{|c|}{ IC do diagnóstico principal } \\
\hline 0 & 4.281 .149 & 76,8 & 761.102 & 84,9 & 2.327 .312 & 76,3 & 1.192 .735 & 73,3 \\
\hline 1 & 860.607 & 15,4 & 93.273 & 10,4 & 486.800 & 16,0 & 280.534 & 17,2 \\
\hline 2 & 404.035 & 7,2 & 41.656 & 4,6 & 220.307 & 7,2 & 142.072 & 8,7 \\
\hline$\geq 3$ & 29.507 & 0,5 & 799 & 0,1 & 16.631 & 0,5 & 12.077 & 0,7 \\
\hline \multicolumn{9}{|c|}{ Fonte de pagamento e processo de cuidado } \\
\hline \multicolumn{9}{|c|}{ Fonte de pagamento da internação } \\
\hline SUS & 3.613 .843 & 64,8 & 226.709 & 25,3 & 1.897 .229 & 62,2 & 1.489 .905 & 91,6 \\
\hline Plano de saúde & 1.723 .472 & 30,9 & 586.291 & 65,4 & 1.020 .770 & 33,5 & 116.411 & 7,2 \\
\hline Particular & 219.050 & 3,9 & 78.966 & 8,8 & 121.085 & 4,0 & 18.999 & 1,2 \\
\hline Filantropia & 18.933 & 0,3 & 4.864 & 0,5 & 11.966 & 0,4 & 2.103 & 0,1 \\
\hline \multicolumn{9}{|l|}{ Utilização de UTI } \\
\hline Não & 5.196 .278 & 93,2 & 864.047 & 96,3 & 2.831 .228 & 92,8 & 1.501 .003 & 92,2 \\
\hline Sim & 379.020 & 6,8 & 32.783 & 3,7 & 219.822 & 7,2 & 126.415 & 7,8 \\
\hline \multicolumn{9}{|c|}{ Tipo de procedimento } \\
\hline Clínico & 3.040 .757 & 54,5 & 410.038 & 45,7 & 1.704 .269 & 55,9 & 926.450 & 56,9 \\
\hline Cirúrgico & 2.534 .541 & 45,5 & 486.792 & 54,3 & 1.346 .781 & 44,1 & 700.968 & 43,1 \\
\hline
\end{tabular}

IC: Índice de Charlson; SUS: Sistema Único de Saúde; UF: Unidade da Federação; UTI: unidade de tratamento intensivo.

* Desempenho melhor que o esperado;

** Desempenho compatível ao esperado;

*** Desempenho pior que o esperado.

Fonte: Ministério da Saúde. Cadastro Nacional de Estabelecimentos de Saúde (CNES), Registro de Planos de Saúde (RPS),

Sistema de Informações Hospitalares do SUS (SIH) e Comunicação de Internação Hospitalar (CIH).

planejamento 1,37,38,39. Já sob a ótica da qualidade da assistência hospitalar, pouquíssimos estudos no Brasil compararam o desempenho por fontes de pagamento 19,33, mas nenhum analisou sua composição e variação segundo arranjos de financiamento dos hospitais. Assim, é difícil a tarefa de estabelecer comparações sobre o tema no país.

De todo modo, chama a atenção o fato de que, dentro das mesmas estruturas físicas, haja diferenças na RMHP entre pacientes SUS e Não SUS, como as verificadas neste estudo. Isso pode indicar que, mesmo estando fisicamente disponíveis nos hospitais, alguns recursos não se encontram ao alcance de pacientes cuja fonte de pagamento é o SUS. A maior proporção de hospitais públicos e de arranjo somente SUS classificados com desempenho pior que o esperado indica a ocorrência de problemas que merecem ser investigados e monitorados. Entre os fatores que influenciariam as disparidades nos resultados de pacientes por fontes de pagamento de suas internações, estudos publicados por pesquisadores dos Estados Unidos discutem diferenças da prática clínica, acesso a tecnologias e procedimentos de alto custo e complexidade, além da indução ao uso de serviços desnecessários, impulsionados por mecanismos de mercado 26,40,41,42. No entanto, vale ressaltar que limitações inerentes ao uso da mortalidade hospitalar global, bem como diferenças na gravidade do caso podem também explicar a variação aqui descrita.

\section{Limites}

Os limites deste estudo relacionam-se a três principais questões: a abordagem da qualidade escolhida, qual seja, a análise do resultado do cuida- 
Figura 2

Razão de mortalidade hospitalar padronizada (observada/esperada - razão O/E), por arranjo de financiamento e fonte de pagamento. Estados de São Paulo e Rio Grande do Sul, Brasil, 2008-2010.

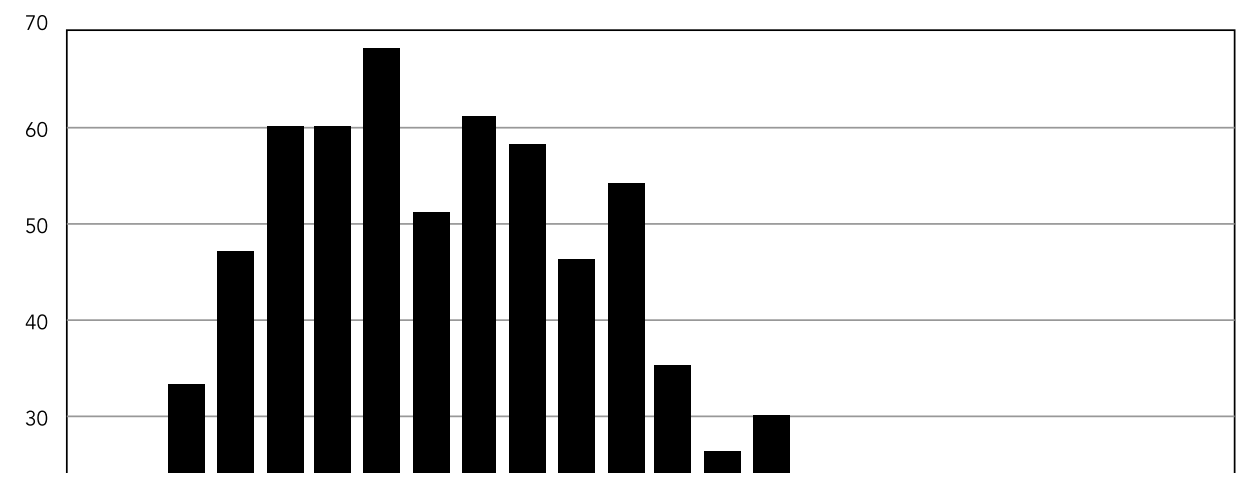

SUS: Sistema Único de Saúde.

Tabela 3

Razão de mortalidade hospitalar padronizada observada/esperada (RMHP O/E) nas internações financiadas pelo Sistema Único de Saúde (SUS) e por planos privados de saúde ocorridas nos hospitais com arranjo misto. Estados de São Paulo e Rio Grande do Sul, Brasil, 2008-2010.

\begin{tabular}{lccc}
\hline \multirow{2}{*}{ Medidas } & \multicolumn{2}{c}{ RMHP O/E } & Total \\
& Desempenho SUS inferior * & Desempenho SUS superior * \\
\hline Hospitais & 305 & 57 & 362 \\
Internações & 2.486 .667 & 283.792 & 2.770 .459 \\
$\quad$ Internações SUS & 1.893 .307 & 210.116 & 2.103 .423 \\
Internações planos & 593.360 & 73.676 & 667.036 \\
Variação O/E & $0,13-2,19$ & $0,10-1,60$ & $0,10-2,19$ \\
O/E SUS & $0,13-3,91$ & $0,04-1,61$ & $0,04-3,91$ \\
O/E planos & $0,02-1,69$ & $0,21-3,00$ & $0,02-3,00$ \\
\hline
\end{tabular}

* Desempenho comparativo do SUS com relação às internações financiadas pelos planos privados de saúde.

Fonte: Ministério da Saúde. Cadastro Nacional de Estabelecimentos de Saúde (CNES), Registro de Planos de Saúde (RPS),

Sistema de Informações Hospitalares do SUS (SIH) e Comunicação de Internação Hospitalar (CIH).

do por meio da mortalidade hospitalar global; a qualidade e o escopo dos dados utilizados; e o recorte do estudo propriamente dito.

Tendo em vista a natureza multidimensional do conceito de qualidade e o fato de a mortalidade hospitalar não mensurar todas as dimensões do cuidado, a abordagem realizada neste estudo deve ser considerada uma aproximação indireta da qualidade ${ }^{43}$. Outra limitação do uso da mortalidade hospitalar está em sua natureza dicotômica, incapaz de medir resultados quali- tativamente diferentes de alta ou morte, como, por exemplo, a redução na qualidade de vida e autonomia dos pacientes. Além disso, a morte resultante de um cuidado hospitalar inadequado pode ocorrer posteriormente à alta hospitalar, como seria o caso de pacientes com alta precoce. As bases de dados utilizadas não permitiram tal análise, o que exigiria a aplicação de metodologias de relacionamento probabilístico de bases de dados, processo complexo e trabalhoso 44,45, já que ainda não há uma chave única capaz de 
vincular os dados registrados nos sistemas brasileiros de internações e mortalidade.

Merecem destaque ainda as questões relacionadas à validade da mortalidade hospitalar associadas à adequação do ajuste de risco, particularmente quando se trabalha com uma gama de causas de morte agrupadas e com informação sobre gravidade do paciente extremamente limitada 17,18 . Nesse sentido, não foi possível identificar as comorbidades ou doenças presentes na admissão, que afetam a condição do paciente e seu prognóstico ${ }^{14}$. Essa é uma limitação que merece a atenção dos gestores da informação nos hospitais, para melhorar o preenchimento do campo destinado ao diagnóstico secundário. Embora no início de 2015 os campos do SIH para o registro dessa informação tenham sido ampliados, os gestores públicos deveriam adotar ações para incentivar esse preenchimento, sobretudo pelas mudanças no perfil demográfico e epidemiológico que resultam na elevada prevalência de múltiplas morbidades crônicas. No setor privado, no que diz respeito à CIHA, o preenchimento observado indica uma necessidade ainda mais contundente de melhoria das informações sobre a produção hospitalar. Também, a impossibilidade de utilizar o tipo de admissão (eletiva/emergência) no ajuste de risco representa uma limitação, que igualmente requer esforço de correção.

Além das limitações inerentes ao uso das bases de dados administrativas, há algumas particularidades das bases brasileiras, em especial a exclusão de informações necessárias, a coleta incompleta ou incorreta de dados, afora os conhecidos problemas de cobertura das internações ocorridas ${ }^{3}$. As questões relacionadas especialmente à cobertura e completude da CIHA afetam a abrangência dos estudos que a utilizam como fonte, limitando os resultados e conclusões alcançados neste estudo a áreas específicas cuja maior qualidade da informação é reconhecida. Todavia, apesar das limitações das bases de dados administrativas, a sua utilização é uma alternativa explorada em muitos países por causa da facilidade de obtenção, baixo custo e abrangência 19,46.

Por fim, há a limitação quanto ao recorte do estudo, visto que aspectos organizacionais e financeiros dos hospitais e do contexto que poderiam contribuir para melhor compreensão dos resultados não fizeram parte do escopo, destacando-se: a disponibilidade de profissionais de saúde e equipamentos por paciente; a rotatividade de leitos; os incentivos ou pressões financeiras; as características de mercado; o investimento em atividades voltadas à qualidade; $\mathrm{o}$ contexto e a cultura institucional; as diferenças regionais; entre outros $4,24,25$.

\section{Considerações finais}

Mesmo com os limites, os resultados deste estudo indicam riscos diferenciados na RMHP em função da fonte de pagamento da internação (SUS ou Não SUS), com desvantagens para os pacientes do SUS, agregando complexidade às análises sobre o tema. Igualmente a grande variação da RMHP, ainda que dentro das três classes de desempenho trabalhadas, e o alto volume de internações realizadas em hospitais classificados no percentil 80 (pior que o esperado) ilustram a importância de um acompanhamento contínuo e aprofundamento da análise desenvolvida. A sobreposição das redes pública e privada e as diferenças de oferta e acesso da população conforme fontes de pagamento constituem um fenômeno específico do sistema de saúde brasileiro, cujos efeitos sobre a qualidade demandam debates não apenas à luz dos métodos de pesquisa em avaliação, mas também de aspectos políticos, econômicos e sociais que o condicionam.

A categorização dos hospitais em arranjos de financiamento proposta para este estudo tem importância para as análises sobre o tema, especialmente porque as iniquidades mais graves parecem ocorrer dentro dos hospitais, quais sejam, naqueles de arranjo Misto. Tal grupo de hospitais deveria ser objeto de estudo e monitoramento, incluindo a investigação de possíveis fatores organizacionais que conduzem os pacientes a processos e resultados do cuidado diversos, mesmo com a disponibilidade física das mesmas estruturas. Esse achado implica discussões que transcendem a gestão do sistema ou o monitoramento da qualidade de serviços, envolvendo questões éticas de extrema relevância social.

Esforços devem ser feitos a fim de tornar o acesso à rede hospitalar e a qualidade do cuidado mais equânimes no país, baseando-se na necessidade de saúde independentemente da fonte de pagamento. Além do alinhamento de esforços públicos e privados às necessidades da população, o monitoramento regular da qualidade desses serviços deve fazer parte do arcabouço de informações usadas no direcionamento de políticas e regulamentações na área da atenção hospitalar no Brasil, aproximando o planejamento em saúde e a prática do cuidado das discussões teóricas e científicas sobre a qualidade dos serviços, em prol de ganhos para a sociedade e para o país. É consenso entre pesquisadores e gestores que atingir determinado padrão de qualidade no sistema de saúde é fundamental não apenas do ponto de vista clínico ou da gestão, mas também para a sua legitimação perante a população. Desse modo, o papel da avaliação e de estratégias de melhoria da qualidade da 
assistência vem ao encontro das preocupações com equidade, acesso, adequação, efetividade e segurança no cuidado, constituindo parte im- portante no projeto de implantação do sistema de saúde desejado para o Brasil.

\section{Colaboradores}

J. P. Machado participou de todas as etapas de desenvolvimento do estudo e elaboração deste artigo. M. Martins participou da concepção, análise dos dados, redação e revisão crítica do documento. I. C. Leite participou da análise dos dados e revisão crítica do documento.

\section{Agradecimentos}

Agradecemos à Agencia Nacional de Saúde Suplementar e ao Conselho Nacional de Desenvolvimento Científico e Tecnológico.

\section{Referências}

1. Santos IS. Evidência sobre o mix público-privado em países com cobertura duplicada: agravamento das iniquidades e da segmentação em sistemas nacionais de saúde. Ciênc Saúde Coletiva 2011; 16:2743-52.

2. Basu S, Andrews J, Kishore S, Panjabi R, Stuckler D. Comparative performance of private and public healthcare systems in low and middle-income countries: a systematic review. PLoS Med 2012; 9:e1001244.

3. Machado JP. O arranjo público-privado no Brasil e a qualidade da assistência hospitalar em São Paulo e no Rio Grande do Sul [Tese de Doutorado]. Rio de Janeiro: Escola Nacional de Saúde Pública Sergio Arouca, Fundação Oswaldo Cruz; 2014.

4. Campbell MJ, Jacques RM, Fotheringham J, Maheswaran R, Nicholl J. Developing a summary hospital mortality index: retrospective analysis in English hospitals over five years. BMJ 2012; 344:e1001.

5. Agency for Healthcare Research and Quality. Guide to inpatient quality indicators: quality of care in hospitals - volume, mortality, and utilization. Version 3.1. http://qualityindicators.ahrq.gov/Down loads/Modules/IQI/V31/iqi_guide_v31.pdf (acessado em 04/Jul/2013).
6. Pitches DW, Mohammed AM, Lilford RJ. What is empirical evidence that hospitals with higher-risk adjusted mortality rates provide poorer quality care? A systematic review of the literature. BMC Health Serv Res 2007; 7:91-8.

7. Canadian Institute for Health Information. HSMR: a new approach for measuring hospital mortality trends in Canada. Ottawa: Canadian Institute for Health Information; 2007.

8. Ben-Tovim D, Woodman R, Harrison JE, Pointer S, Hakendorf P, Henley G. Measuring and re porting mortality in hospital patients. http:// www.aihw.gov.au/WorkArea/DownloadAsset. aspx?id=6442457122 (acessado em 22/Jan/2013).

9. Fung V, Schmittidiel JA, Fireman B, Meer A, Thomas S, Smider N, et al. Meaningful variation in performance: a systematic review. Med Care 2010; 48:140-8.

10. Chassin MR, Loeb JM, Schmaltz SP, Wachter RM. Accountability measures: using measurement to promote quality improvement. N Engl J Med 2010; 363:683-8.

11. Groene O, Skau JKH, Frølich A. An international review of projects on hospital performance assessment. Int J Qual Health Care 2008; 20:162-71. 
12. Goodacre S, Campbell M, Carter A. What do hospital mortality rates tell us about quality of care? Emerg Med J 2015; 32:244-7.

13. Iezzoni LI. Risk adjustment for measuring health care outcomes. 3rd Ed. Ann Arbor: Health Administration Press; 2003.

14. Sharabiani MTA, Aylin P, Bottle A. Systematic review of comorbidity indices for administrative data. Med Care 2012; 50:1109-18.

15. Shahian DM, Iezzoni LI, Meyer GS, Kirle L, Normand SLT. Hospital-wide mortality as a quality metric: conceptual and methodological challenges. Am J Med Qual 2012; 27:112-23.

16. Mackenzie SJ, Goldman DA, Perla RJ, Parry GJ. Measuring hospital-wide mortality - pitffals and potential. J Healthc Qual 2016; 38:187-94.

17. van Gestel YRBM, Lemmens VEPP, Lingsma HF, Hingh IHJT, Rutten HJT, Coebergh JWW. The hospital standardized mortality ratio fallacy: a narrative review. Med Care 2012; 50:662-7.

18. Thomas JW, Hofer TP. Research evidence on the validity of risk-adjusted mortality rate as a measure of hospital quality of care. Med Care Res Rev 1998; 55:371-404.

19. Martins M, Blais R, Leite IC. Mortalidade hospitalar e tempo de permanência: comparação entre hospitais públicos e privados na região de Ribeirão Preto, São Paulo, Brasil. Cad Saúde Pública 2004; 20:268-82.

20. Shahian DM, Wolf RE, Iezzoni LI, Kirle L, Mormand SLT. Variability in the measurement of hospital-wide mortality rates. N Engl J Med 2010; 363: 2530-9.

21. Jarman B, Gault S, Alves B, Hider A, Dolan S, Cook A, et al. Explaining differences in English hospital death rates using routinely collected data. BMJ 1999; 318:1515-20.

22. Jarman B, Pieter D, van der Veen AA, Kool RB, Aylin P, Bottle A, et al. The hospital standardized mortality ratio: a powerful tool for Dutch hospitals to assess their quality of care? Qual Saf Health Care 2010; 19:9-13.

23. Institute for Healthcare Improvement. Move your dot: measuring, evaluating, and reducing hospital mortality rates (Part 1). Boston: Institute for Healthcare Improvement; 2003. (Innovation Series 2003).

24. Brand CA, Barker AL, Morello RT, Vitale MR, Evans SM, Scott IA, et al. A review of hospital characteristics associated with improved performance. Int J Qual Health Care 2012; 24:483-94.

25. Eggleston K, Shen YC, Lau J, Schmid CH, Chan J. Hospital ownership and quality on care: what explains the different results in the literature? Health Econ 2008; 17:1345-62.

26. Spencer CS, Gaskin DJ, Roberts ET. The quality of care delivered to patients within the same hospital varies by insurance type. Health Aff 2013; 32:1 731-9.

27. Weissman JS, Vogeli C, Levy DE. The quality of hospital care for Medicaid and private pay patients. Med Care 2013; 51:389-95.
28. Machado JP, Martins ACM, Martins MS. Avaliação da qualidade do cuidado hospitalar no Brasil: uma revisão sistemática. Cad Saúde Pública 2013; 29:1063-82.

29. Martins M. Uso de medidas de comorbidades para predição de risco de óbito em pacientes brasileiros hospitalizados. Rev Saúde Pública 2010; 44:448-56.

30. Quan H, Sundararajan V, Halfon P, Fong A, Burnand B, Luthi JC, et al. Coding algorithms for defining comorbidities in ICD-9-CM and ICD-10 administrative data. Med Care 2005; 43:1130-9.

31. Aylin P, Bottle A, Majeed A. Use of administrative data or clinical databases as predictors of risk of death in hospital: comparison of models. BMJ 2007; 334:1044

32. Noronha JC, Martins M, Travassos C, Campos MR, Maia P, Panzzuti R. Aplicação da mortalidade hospitalar após a realização de cirurgia de revascularização do miocárdio para monitoramento do cuidado hospitalar. Cad Saúde Pública 2004; 20 Suppl 2:S322-30.

33. Gomes AS, Klück MM, Fachel JMG, Riboldi J. Fatores associados à mortalidade hospitalar na rede SUS do Rio Grande do Sul, em 2005: aplicação de modelo multinível. Rev Bras Epidemiol 2010; 13:533-42.

34. Noronha JC, Travassos C, Martins M, Campos MR, Maia P, Penezzuti R. Avaliação da relação entre volume de procedimentos e a qualidade do cuidado: o caso de cirurgia coronariana no Brasil. Cad Saúde Pública 2003; 19:1781-9.

35. Travassos C, Noronha J, Martins M. Mortalidade hospitalar como indicador de qualidade: uma revisão. Ciênc Saúde Coletiva 1999; 4:367-81.

36. Barisonzo R, Wiedermann W, Unterhuber M, Wiedermann CJ. Length of stay as risk factor for inappropriate hospital days: interaction with patient age and co-morbidity. J Eval Clin Pract 2013; 19: 80-5.

37. Medici AC. Sistemas de financiamento e gestão hospitalar: uma aplicação ao caso brasileiro. In: Vecina Neto G, Malik AM, organizadores. Gestão em saúde. Rio de Janeiro: Editora Guanabara Koogan; 2011. p. 50-72.

38. La Forgia GM, Couttolene BF. Desempenho hospitalar no Brasil: em busca da excelência. São Paulo: Singular; 2009.

39. Vecina Neto G, Malik AM. Tendências na assistência hospitalar. Ciênc Saúde Coletiva 2007; 12: 825-39.

40. Epstein AJ, Ketcham JD, Rathore SS, Groeneveld PW. Variations in the use of an innovative technology by payer: the case of drug-eluting stents. Med Care 2012; 50:1-9.

41. Hadley J, Steinberg EP, Feder J. Comparison of uninsured and privately insured hospital patients: condition on admission, resource use, and outcome. JAMA 1991; 265:374-9.

42. Mulley AG. Inconvenient truths about supplier induced demand and unwarranted variation in medical practice. BMJ 2009; 339:b4073. 
43. Donabedian A. Explorations in quality assessment and monitoring. Volume I. The definition of quality and approaches to its assessment. Ann Arbor: Health Administration Press; 1980

44. Pinheiro RS, Silva JRN, Lima CRA, Coeli CM. Cobertura da Comunicação de Informação Hospitalar e Ambulatorial (CIHA) utilizando os partos registrados no Sistema de Informações sobre Nascidos Vivos, Brasil, 2006 a 2009. Cad Saúde Pública 2012; 28:991-7.

\begin{abstract}
In Brazil, the combined presence of public and private interests in financing and provision of healthcare services stands out clearly in hospital care. Financing arrangements adopted by hospitals (the public Brazilian Unified National Health System - SUS and/or health plans and/or out-of-pocket payment) can affect quality of care. Studies have analyzed the hospital standardized mortality ratio (HSMR) in relation to quality improvements. The objective was to analyze HSMR according to source of payment for the hospitalization and the hospital's financing arrangement. The study analyzed secondary data and causes that accounted for $80 \%$ of hospital deaths. HSMR was calculated for each hospital and payment source. Hospitals with worse-than-expected performance (HSMR $>1$ ) were mostly large public hospitals. HSMR was higher in the SUS, including between admissions in the hospital. Despite the study's limitations, the findings point to inequalities in results of care. Efforts are needed to improve the quality of hospital services, regardless of the payment sources.
\end{abstract}

Quality of Health Care; Outcome Assessment (Health Care); Public-Private Sector Partnerships; Hospital Mortality
45. Moreira ML, Novaes HMD. Internações no sistema de serviços hospitalares, SUS e não SUS: Brasil, 2006. Rev Bras Epidemiol 2011; 14:411-22.

46. McGlynn EA. Measuring clinical quality and appropriateness. In: Smith PC, Mossialos E, Papanicolas I, Leatherman S, editors. Performance measurement for health system improvement: experiences, challenges and prospects. Cambridge: Cambridge University Press; 2009. p. 87-113.

\section{Resumen}

En Brasil, la convivencia público-privada en la financiación y en la prestación del cuidado empieza a definirse nítidamente en la asistencia hospitalaria. Los acuerdos de financiación adoptados por los hospitales (Sistema Único de Salud-SUS y/o planes de salud y/o pago particular) pueden afectar a la calidad del cuidado. Algunos estudios buscan asociar la razón de mortalidad hospitalaria padronizada (RMHP) a mejorías en la calidad. El objetivo fue analizar la RMHP según la fuente de pago del internamiento y acuerdos de financiación del hospital. Se analizaron datos secundarios y causas responsables de un $80 \%$ de los óbitos hospitalarios. La RMHP se calculó para cada hospital y fuente de pago. Los hospitales con un desempeño peor que el esperado $(R M H P>1)$ fueron mayoritariamente públicos y con un mayor número de pacientes. La RMHP en los internamientos SUS fue superior, incluyendo internamientos en el mismo hospital. A pesar de los límites, los hallazgos indican inequidades en el resultado del cuidado. Son necesarios esfuerzos dirigidos a la mejoría de la calidad de servicios hospitalarios, independientemente de las fuentes de pago de los mismos.

Calidad de la Atención de Salud; Evaluación de Resultado (Atención de Salud); Asociaciones entre el Sector Público y el Privado; Mortalidad Hospitalaria
Recebido em 15/Jul/2015

Versão final reapresentada em 21/Set/2015 Aprovado em 18/Fev/2016 